\title{
Shaping embryonic development
}

\author{
The growing intersection between chemical tools and principles and developmental biology is providing new \\ insights into the molecular-level details of developmental processes.
}

Developmental biology has largely relied on molecular biology and genetic approaches to identify and perturb developmental regulators in model organisms including worms, zebrafish, and mice. However, there is a growing need for more reversible and precise ways to perturb developmental pathways. Recent efforts have also focused on understanding the chemical basis of developmental signaling and on applying this information to create artificial systems that mimic developmental properties. Chemical biology is well suited to developing improved tools that enable the manipulation of developmental processes with greater spatiotemporal resolution and further elucidating the chemical logic of cell-tocell signaling. In this themed issue, we offer a collection of pieces focusing on this intersection of chemical approaches with developmental biology and highlighting how collaborations at this interface may reveal new insights.

Although some of the relevant genes and signaling pathways involved in embryonic development have been identified, the development of organisms such as the nematode Caenorhabditis elegans is responsive to environment signals. Under low-nutrient conditions, worms enter a developmentally arrested stress-protective stage called the "dauer," controlled through the secretion of pheromones that are fatty acid derivatives of the L-sugar ascarylose. Butcher (Perspective, p. 577) describes recent advances using NMR and activity-guided fractionation to characterize the enzymes required for dauer pheromone synthesis. In addition, recent findings have identified hybrid polyketide-nonribosomal peptides called nemamides (Nat. Chem. Biol. 12, 770-772, 2016) that promote larval survival during starvation arrest and a class of phosphorylated glycosphingolipids called phosphoethanolammine glucosylceramides (PECGC) that mobilize an internal sterol pool (Article, p. 647).

Plants, like worms, require a combination of environmental cues and small-molecule hormones for growth and development. Identification of the receptors and effectors for plant hormones such as auxin, brassinosteroids and strigolactones (SLs) has provided insights into how these hormones are received and has enabled the elucidation of their biological functions (Nat. Chem. Biol. 5, 267, 2009).
As SL is also recognized by parasitic plants to stimulate their germination, the balance between SL perception by the host plant and parasite is critical. Lumba, Holbrook-Smith and McCourt (Review, p. 599) discuss recent findings, using fluorescent probes to image SL perception by the plant parasite Striga and small-molecule inhibitors that block strigolactone signaling in plants and parasites (Nat. Chem. Biol. 12, 724-729, 2016).

Increased knowledge about the biosynthesis of developmental signaling molecules has revealed requirements for posttranslational modification of either the ligands or the receptors. In particular, Wnt proteins require fatty acylation by the $O$-acyltransferase porcupine to regulate Wnt intracellular trafficking (Nat. Chem. Biol. 12, 60-69, 2016). The crystal structure of the Notch receptor with the Jagged ligand (News and Views, p. 570) has revealed conformational changes that explain how glycosylation of Notch alters its responsiveness to the Delta and Jagged ligands

The ability of small molecules to alter developmental signaling pathways with reversible and temporal control has been useful in promoting the differentiation of pluripotent stem cells into various cellular lineages.

However, because many of the cellular targets that alter stem cell reprogramming remain uncharacterized, phenotypic screening can be a valuable discovery approach to identify novel stem cell regulators. Waldmann, Scholer and Ursu (Commentary, p. 560) discuss the current challenges for stem cell phenotypic screening, such as the need to ensure the reproducible growth and characterization of stem cell lines and the requirement for extensive validation methods to identify the relevant specific molecular targets. A recent example that combines expertise in stem cell and chemical biology was the identification of Epiblastin A (Cell. Chem. Biol. 23, 494-507, 2016) as a promoter of mouse epiblast stem cell reprogramming into mouse embryonic stem cells. This example highlights how improved collaborations between stem cell researchers and chemical biologists could strengthen chemical probe discovery in stem cells by providing strong biological and technical synergy.

Precise cell-to-cell communication is required for many developmental processes such as concerted migration. Although some aspects of this intercellular network have been gleaned from genetic and molecular studies, Gartner, Prescher and Lavis (Commentary, p. 564) propose that chemical tools are needed to detect, disrupt and reconstitute these intracellular networks using a combination of imaging reagents, cell-type-specific pharmacology and synthetic extracellularmatrix components. One example of this type of technology is an optogenetic and bioluminescent system that detects oscillatory gene expression transfer between cells with spatiotemporal resolution (Research Highlights, p. 569).

Although optogenetic approaches have been used in cell culture to modulate gene regulation (Nat. Chem. Biol. 12, 381, 2016), their potential to modulate developmental processes with spatiotemporal precision remains unfulfilled. Kowalik and Chen (Perspective, p. 587) describe some recent examples in which applications of optochemical and optogenetic systems in developmental organisms have revealed new insights. In particular, the use of photoactivable morpholino oligonucleotides targeting the transcription factor Tbx16 combined with transcriptome analysis revealed a role of posterior hox gene repression in mesodermal progenitor cells (Nat. Chem. Biol. 12, 694-701, 2016).

Despite the progress made in addressing developmental questions with chemical tools, there are still some unresolved issues. Organisms such as worms and fly embryos are not amenable to chemical modification because of issues with uptake (Nat. Chem. Biol. 6, 549-557, 2010), and there may be some reluctance among developmental biologists to use chemical tools given the legacy of molecular biology and genetics. To address this issue, researchers with dual expertise in developmental biology and chemistry are needed, but they remain a small minority, as there are limited training opportunities in both disciplines. In lieu of that, more collaboration between chemical biologists and embryologists are needed to provide complementary expertise and to remove current technical and cultural barriers to progress. At Nature Chemical Biology, we hope that the pieces in this issue will inspire and motivate more partnerships that will help drive the field forward and support the growing intersection between chemical biology and developmental biology. 